\title{
Ueber eine neue vereinfachte Construction der sogenannten Achsenzugzangen.
}

\author{
Von \\ Dr. C. Breus, \\ Assistent an Professor Gustav Braun's Klinik in Wien.
}

(Mit 9 Holzschnitten.)

Die Frage nach der Reformfähigkeit und Reformbedürftigkeit des Forceps beschäftigt, seit sie durch Tarnier ${ }^{1}$ ) neuerlich angeregt worden, die Geburtshelfer aller Länder. Seit er den Anstoss gegeben sind die Stimmen, die sich pro und contra erheben, nicht wieder verstummt. Auch neue Vorschläge in der von ihm eingeschlagenen Richtung hat man gebracht, da man, ohne sich mit Tarnier's Instrument zufrieden zu geben, die Sache doch nicht fallen lassen wollte, und Tarnier selbst hat seit seiner ersten Publication bereits mehrere neue Modelle construirt.

Eine ausführliche Schilderung der ganzen Bewegung vor und seit Tarnier hat $\mathrm{Sänger}^{2}$ ) in diesem Archive geliefert, so dass ich, um weitläufige Wiederholungen zu vermeiden, auf dessen verdienstliche Arbeit verweisen kann.

Professor Gustav Braun hat Tarnier's Instrument sofort

1) „Description de deux nouveaux forceps.“ Paris 1877. - „Discussion relative au nouveau forceps", Annales de Gynécologie 1877.

2) „Ueber Zangen mit Zugapparaten $u$. achsengemässe Zangenextraction. Die Zangen von Tarnier, ihre Vorläufer und Modificationen. Anpassung der deutschen Zangen an deren Principien", von Dr. M. Sänger. Dieses Archiv, Bd. XVII, S. 382.

Die in dieser Arbeit empfohlene Sänger'sche ,Zugriemenzange“ hat wohl hauptsächlich deshalb keine allgemeine Verbreitung gefunden, weil sie, wie Sänger selbst andeutet, zu sehr den Charalkter einer Improvisation trägt. 
nach dessen Bekanntwerden an seiner Klinik versucht und sein Urtheil in $\mathrm{Nr}, 24$ und 25 der Wiener medicinischen Wochenschrift 1880 dahin abgeben, dass das Instrument einzelne wesentliche Vortheile habe, die aber durch verschiedene Mängel wieder abgeschwächt werden. Weit davon entfernt, die Fehler anzuerkennen, welche Tarnier unserem gewöhnlichen Forceps vorwirft, hat Prof. Gust. Braun doch den Achsenzugzangen seine Aufmerksamkeit zugewendet. Da er die Richtigkeit der Grundidee Tarnier's bestätigt gefunden, so liess er die Versuche mit dem Instrumente fortsetzen und hat ebenso den von A. R. Simpson 1) im gleichen Sinne construirten Axis-traction forceps an seiner Klinik in versuchsweise Verwendung gebracht.

So kam ich in die Lage, mit diesen beiden besten Constructionen von Achsenzugzangen zahlreiche Operationen zu sehen oder selbst auszuführen. Dabei gewann ich die Ueberzeugung, dass das Princip dieser Zangen, nach welchem dieselben mit geringerem Kraftverluste zu operiren erlauben, ebenso seine thatsächliche Bestätigung finde, wie es theoretisch einleuchtend ist.

Dass die Aufnahme, welche Tarnier gefunden, eine so getheilte, erklärt sich hauptsächlich aus zwei Momenten. Erstens ist die technische Ausführung seiner Idee bei Tarnier zu complicirt gerathen und seine Zange vom bewährten Typus des, Forceps ordinaires' zu sehr abgewichen, so dass Schwerfälligkeit und Unverlässlichkeit in, der Handhabung neben anderen Nachtheilen, die aus einzelnen Eigenthümlichkeiten der Construction entspringen, viele Kritiker verstimmen mussten.

Und zweitens hat Tarnier in der Beurtheilung des gewöhnlichen Forceps und der Leistungsfähigkeit seines Instrumentes alles Maass verloren. Etwas mehr Zurückhaltung in dieser Hinsicht hätte ihm gewiss manchen Widerspruch erspart.

Er will den alten Forceps ganz verdrängen. Auch dort, wo dieses Instrument ganz unübertrefflich fungirt am sogenannten zangenrechten, rotirt am Beckenboden stehenden Schädel, will Tarnier sein complicirtes Instrument an Stelle des einfachen setzen. Also auch, wo das Bedürfniss nach einem neuen Instrumente gar nicht besteht.

Andererseits wieder empfiehlt Tarnier seinen modificirten Forceps auch für Fälle, wo besonnene Geburtshelfer längst die

1) „On axis-traction forceps." Edinburgh 1880. 
Wendung der Zangenoperation vorziehen gelernt haben und letztere streng verpönen. Sogar den noch beweglich über dem Beckeneingange stehenden Schädel will Tarnier mit seiner Zange fassen.

So opfert er seinem Instrumente auch alle theoretischen, die Indication und Anwendbarkeit betreffenden Erfahrungen, die wir dem alten Instrumente danken, ebenso wie er alle die vortheilhaften Einzelheiten der einfachen und bewährten Construction der gewöhnlichen Zange aufgab.

An seinen früheren Modellen sind die Fenster der Löffel fast mehr das einzige, was in dem Instrumente noch den Forceps erkennen lässt. Daher kommt es, dass die Gegner das Instrument ein Mal überflüssig finden, das andere Mal demselben nicht jene Leistungsfähigkeit zuerkennen, welche ihm sein Erfinder zuschreibt, und sagen, dass man ebensoviel mit dem alten Forceps und zum Theil sogar noch Besseres leisten kann.

Für die einfachen Zangenoperationen am rotirten zangenrechten Schädel ist Tarnier's Forceps in der That überflüssig. In diesen Fällen ist das gewöhnliche Instrument nicht nur unübertrefflich, sondern ist wegen der Einfachheit seiner Handhabung dem umständlichen Tarnier'schen Apparate entschieden vorzuziehen.

Der Vortheil der Kraftersparniss, den Tarnier bietet, ist in solchen Fällen illusorisch. In den extremen Fällen aber, wo der Schädel beweglich, noch nicht im Beckeneingange steht, und wo aus in allen geburtshülflichen Lehrbüchern genugsam erörterten Gründen die Anwendung der Zange unstatthaft ist und gegenüber dem minder gefährlichen Verfahren der Wendung zurücktreten muss, hat Tarnier seinem Instrumente nahezu Unmögliches zugemuthet. Es kann uns daher nicht überraschen, wenn wir von Operateuren, die den neuen Forceps in solchen Fällen versuchten, viele Misserfolge verzeichnet finden.

Die Fälle, wo ein Instrument in Tarnier's Sinne construirt am Platze wäre, sind jene glücklicherweise nicht häufigen, wo der Schädel bereits genügend fixirt mit seinem Centrum nur wenig über dem Beckeneingange steht ${ }^{1}$ ) oder mit seinem grössten

1) Was darüber hinausgeht, eine Operation am unfixirten, beweglich über dem Beckeneingange stehenden Schädel, misslingt gerade nicht immer, bleibt aber doch stets ein Wagniss. 
Umfange die Conjugata schon passirt hat, die Wendung nicht mehr ohne grösste Gefahr möglich, das noch lebende Kind aber nicht ohne weiteres der Perforation verfallen darf und die Geburt doch vollendet werden muss.

Da stellen sich der Extraction mit dem Forceps oft grössere Schwierigkeiten entgegen, welche das sonst so segensvolle Instrument zu einem recht gefährlichen machen. Die Construction der gewöhnlichen Zange verursacht hier einen grösseren Kraftverlust und daher einen grösseren Kraftaufwand. Je grösser aber die Kraft, mit welcher operirt wird, desto grösser wird auch die Gefahr für Mutter und Kind.

In solchen Fällen von atypischen Zangenoperationen, die sich weder durch die Wendung noch durch die Kraniotomie immer umgehen lassen, bietet Tarnier's Forceps dem Operateur einen unschätzbaren Vortheil, nämlich die Möglichkeit, mit geringerem Kraftaufwande zu arbeiten, weil weniger Kraft verloren geht. ${ }^{1}$ )

Am wenigsten die Schonung des Operateurs oder die äussere Würde der Operation sind es, um derentwillen dieser Kraftgewinn wünschenswerth erscheint. Vielmehr die grössere Sicherheit und Ruhe, mit welcher bei geringerer Muskelaction operirt werden kann. Die genauere, verlässlichere Controle der Kraftwirkung und die geringere Gefährdung der dem Zuge und Drucke ausgesetzten Gewebe. Denn die Kraft, welche bei schweren Zangenoperationen erfolglos bleibt, geht ja nur für die Fortbewegung des Schädels verloren, äussert sich aber in sehr bedenklicher Weise um so mehr durch Druck an den Angriffspunkten am Schädel und an der Beckenwand. Mit je geringerem Kraftaufwande daher solche Operationen ausgeführt werden können, desto mehr verlieren sie von ihrer Gefährlichkeit. In dieser

1) Der von mehreren Kritikern des Instrumentes erhobene Vorwurf, dass man mit demselben eine enorme Kraft aufbringen könne, beruht auf einen offenbaren Missverständnisse.

Ziehen kann man an dem gewöhnlichen Instrumente ebenso stark.

Die Kraft, mit der am Forceps Tarnier gezogen wird, multiplicirt sich ja nicht vermöge der Construction des Instrumentes, wie etwa bei dem Rollensystem eines Flaschenzuges. Es wird nur ein grösserer Theil der angewendeten Kraft für den eigentlichen Zweck, die Fortbewegung des Schädels, wirksam, statt sich in falseher Richtung nachtheilig zu äussern und so für die Extraction verloren zu gehen. 
Hinsicht also darf die Leistung Tarnier's nicht unterschätzt werden.

Leider aber wird dieser unleugbare Vorzug der Achsenzugzangen wieder wettgemacht durch Nachtheile, die sich aus der complicirten Construction dieser Apparate ergeben.

So erklären sich die verschiedenen bald zustimmenden, bald absprechenden Urtheile. Nur eine von mehreren Seiten abgegebene Ansicht, dass alle derartigen Versuche, die Zange zu verbessern, einfach überflüssig seien, da man mit dem gewöhnlichen Forceps in allen Fällen sein Auslangen finden könne, erscheint mir gänzlich ungerechtfertigt und inconsequent. Weil man etwas gut zu Stande bringt, ist der Versuch, es besser zu machen, überflüssig?

Nun kann man aber nicht einmal die Leistungen des Forceps so kurzweg als solche hinstellen, mit welchen man sich zufrieden geben kann. Sondern man muss wohl trennen zwischen einfachen und schweren Zangenoperationen, typischen und atypischen, wie sich $Z_{w}$ eifel ausdrückt.

So ideal die Verwendbarkeit des Forceps am sogenannten zangenrechten Schädel ist, so ganz anders gestaltet sich die Sache, wenn man am höher stehenden Schädel zu operiren hat. Dann wird das Instrument unter Bedingungen in Anwendung gebracht, denen sein Bau nicht mehr entspricht, denen es sich nur wohl oder übel accommodiren muss, und aus der ,unschädlichen Kopfzange" wird ein Instrument, das mit der grössten Vorsicht gehandhabt werden muss. Der Forceps in seinen bei uns gebräuchlichsten Formen der J. Simpson'schen und der Nägele'schen Zange ist eben seiner ganzen Construction nach bestimmt zur Application an den rotirt im Beckenausgange stehenden, den ,zangenrechten“ Schädel. An diesen angelegt gilt der Forceps als ungefährlich, und solche Operationen als so harmlos, dass man ihre Indication stillschweigend nicht einmal als einer so besonders peinlichen Motivirung bedürftig gelten lässt.

Spiegelberg konnte in seinem Lehrbuche sogar Zeitersparniss für den Geburtshelfer als bestimmend zu einer solchen Operation hinstellen und von Luxusoperationen mit dem Forceps sprechen. tionen.

Weit ernster ist der Charakter der atypischen Zangenopera-

Allerdings auch, wenn der Schädel eben erst in das Becken 
eingetreten, vermag der einfache Forceps in den Händen eines tüchtigen Operateurs seiner erschwerten Aufgabe gerecht zu werden. Die Extraction des hochstehenden Schädels mit der gewöhnlichen Zange wird von allen Geburtshelfern bei zwingenden Anlässen ausgeführt. Aber alle Gynäkologen warnen zur äussersten Vorsicht im Gebrauche der Zange unter solchen Verhältnissen, und man schränkt daher die Indication dieser hohen Zangenoperationen überall auf das Nothwendigste ein. Nur dann, wenn die Wendung nicht mehr ausführbar und man vor der Perforation des lebenden Kindes doch noch zurïckschreckt und kein weiteres Zuwarten mehr zulässig ist, nur dann ist der Versuch, den hochstehenden Schädel mit der Zange zu extrahiren, gestattet. Aber auch nur der Versuch.

Bei Missverhältniss zwischen Schädel und Becken, welches schon in seinen geringeren Graden so oft zu Complicationen führt, die eine operative Beendigung der Geburt erheischen, giebt C. von Braun der Zange sogar nur den Werth eines Untersuchungsinstrumentes, indem er dringendst davor warnt, unter solchen Verhältnissen eine Zangenoperation, wenn sie auf Schwierigkeiten stösst, forciren $\mathrm{zu}$ wollen. Man soll dann nur Probezüge machen, um die Accommodationsfähigkeit des Schädels zu prüfen, und fällt diese Probe ungünstig aus, die Zange lieber wieder abnehmen.

Der betonte Unterschied zwischen typischen und atypischen Zangenoperationen ist also ein sehr augenfälliger. Nur für die ersteren Fälle ist eben der Forceps geschaffen. In den anderen bedient man sich seiner nur deshalb, weil man kein passenderes Instrument hat. Versuche, welche den Zweck haben, die Zange auch für solche Fälle geeigneter zu machen, können daher durchaus nicht für überflüssig erklärt werden. Sie haben ihre vollste Berechtigung, denn sie entsprechen einem Bedürfniss. Nur der Excess Tarnier's in der Verurtheilung des „Forceps ordinaire“ und der zu sanguinisch gefassten Mission seines modificirten Instrumentes konnte solchen Widerstand herausfordern. Denn dass wir nicht einmal bei Application der Zange am im Beckenausgange stehenden Schädel in der Richtung der Beckenachse ziehen können, das glauben wir Tarnier nicht, selbst wenn seine Zeichnungen, durch welche er diese Behauptung annehmbar zu machen sucht, minder bestreitbar wären, so dass man ihnen mehr Beweiskraft zugestehen müsste. 
Die Gefährlichkeit schwererer Zangenoperationen und die schlechte Statistik derselben hat ihren Grund hauptsächlich in der zur Extraction nothwendigen Kraft und dem Widerstande, den diese findet. Die Druckeffecte am kindlichen Schädel und an den Wandungen des Geburtskanales sprechen dafür. Warum so viel Kraft zur Extraction des hochstehenden Schädels nöthig ist, erklärt Tarnier durch folgende Zeichnung.

Fig. 1 .

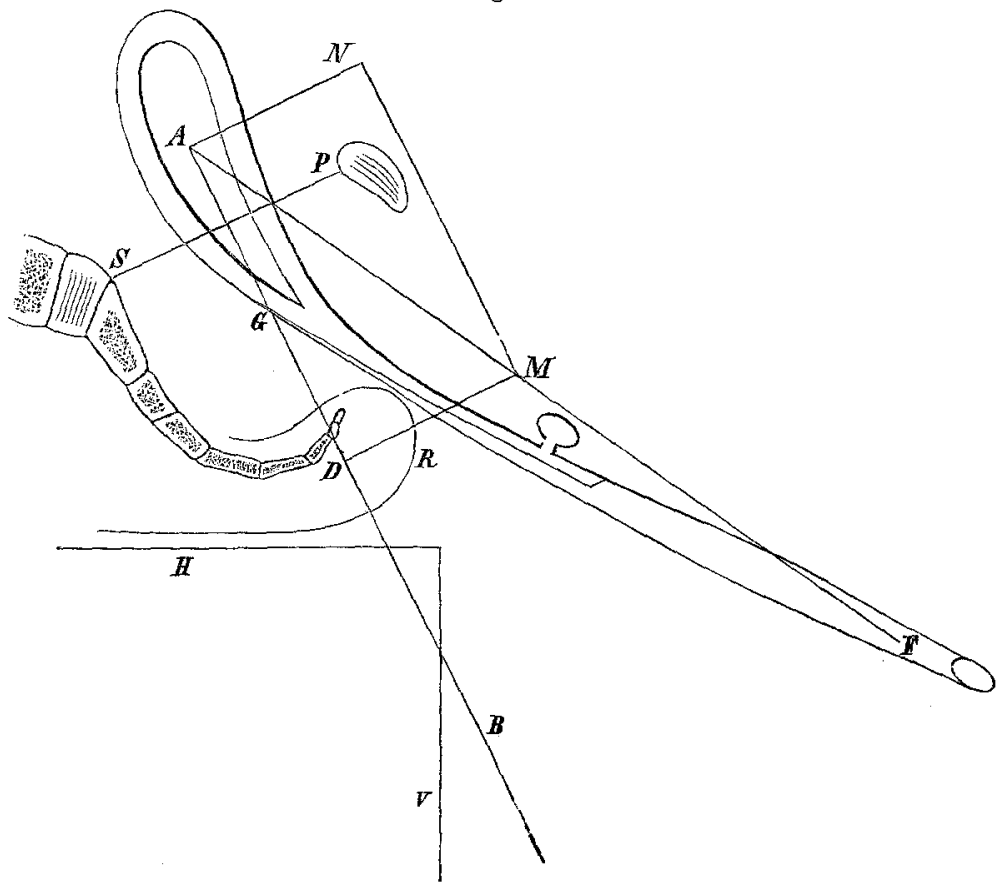

(Aus Tarnier's Monographie, Fig. 1: Forceps ordinaire appliqué au-dessus du détroit supérieur.)

Beim hochstehenden Schädel fällt die Zugrichtung am „Forceps ordinaire" nicht mit der Krümmungslinie des Beckenkanales, in. der der Schädel sich fortbewegen kann, zusammen. Die Zugkraft zerfällt daher in zwei Componenten. Eine $(A D)$ bewegt den Schädel in der Führungslinie fort. Die andere $(A N)$ presst ihn nur gegen die Symphyse an und erschwert so die Extraction.

Wenn auch die Ziffern Tarnier's nicht zutreffen ${ }^{1}$ ), so viel geht doch hervor, dass durch diese Componente Kraft verloren

1) Die Grössen, die Tarnier für diese Componenten mit 34 und 20 Kilogrammen bei einer Zugkraft von 40 Kilo nach dieser Zeichnung ausArchiv f. Gynäkologie. Bd. XX. Hft. 2. 
geht, d. h. nur den nachtheiligen Druck und den Widerstand vermehrt, aber nichts für die Fortbewegung des Schädels leistet, die ja in dieser Richtung durch die vordere Beckenwand behindert ist. Die Folge davon ist, dass man die Kraft, mit welcher am Forceps gezogen wird, verstärken muss. Dadurch fällt die schädliche Componente dann noch grösser aus, bis endlich auch die andere so gross wird, dass der Schädel dem Zuge folgen muss.

Je mehr aber die Muskeln des Operateurs oder gar dessen Körpergewicht (was freilich nicht erlaubt, aber doch gewiss nur zu oft der Fall ist) bei den Tractionen in Rechnung kommen, desto geringer wird die Sicherheit in der Beherrschung der angewendeten Kraft, desto mehr gewinnt die Operation den Charakter auf Biegen oder Brechen ( $\mathrm{Z}$ weifel) und die traurigen Folgen bleiben nicht aus.

Tarnier stellte daher in seiner Eingangs citirten Abhandlung die drei bekannten Anforderungen ${ }^{1}$ ) an eine Zangenmodification, um mit geringerem Kraftaufwande ziehen zu können.

1) „De permettre à l'opérateur de pouvoir toujours tirer suivant l'axe du bassin, quelle que soit la situation de la tête dans la filière pelvienne;

2) de laisser à la tête foetale assez de mobilité pour qu'elle puisse suivre librement la courbure du bassin;

rechnet, sind nicht richtig. Denn wir ziehen ja nicht, wie er uns zumuthet, in der Richtung $A M$, sondern nähern diese doch mehr der Linie $A D$. Auch ist in Tarnier's Zeichnungen das Verhältniss des Forceps zum Becken verzeichnet. Das Becken zu klein, der Forceps zu gross. An dieser Zeichnung z. B. kommt ja, obwohl die Löffel die Ebene des Beckeneinganges weit überragen, das Griffende des Forceps fast in noch grössere Entfernung rom Becken zu liegen, als das Knie der Frau, wenn man sich eine untere Extremität der Grösse des Beckens entsprechend in der Zeichnung ergänzt. Ein solches Ungethüm ist unser „Forceps ordinaire“" doch nicht.

1) In seiner jüngsten Publication (Annales de Gynécologie, Juin. 1882) präcisirt Tarnier dieselben etwas anders, indem er sagt: "Mon forceps repose sur les quatre principes suivants:

a) tirer directement suivant l'axe du bassin;

b) appliquer la traction le plus près possible du centre de la tête;

c) laisser à la tête saisie par le forceps la mobilité, qu'elle a dans l'accouchement naturel, soit pour se fléchir ou se défléchir, soit pour pivoter autour de l'axe fictif du bassin;

d) avoir une aiguille indicatrice qui montre à chaque instant à l'opérateur les divers mouvements exécutés par la tête foetale et qui le guide dans la direction qu'il doit donner à ses tractions." 
3) de présenter une aiguille indicatrice montrant à l'accoucheur la direction qu'il doit donner à ses tractions pour qu'elles soient irréprochables."

Hinsichtlich des ersten Punktes unterschätzt Tarnier die gewöhnliche Zange, wie ihm seither vielfach von $\mathrm{Pajot^{1 }}$ ) bis Lahs ${ }^{2}$ ) vorgehalten wurde. Punkt 1 und 3 beruhen auf Tarnier's zu weit gehender Vorstellung von der Wichtigkeit des Zuges in der Richtung der Beckenachse. Im zweiten von Tarnier dagegen nicht genug hervorgehobenen Punkte gipfelt der Fortschritt, den Tarnier's Constructionen bedeuten. Wenn nur diese Bedingung erfüllt ist, das Instrument dem Schädel genügende Freiheit für seine Bewegung durch das Becken lässt, dann ist die grössere oder geringere Uebereinstimmung der Zugrichtung mit der Beckenachse nebensächlich.

Die bei der spontanen Geburt wirkende Triebkraft der Uteruscontractionen hat ja auch nichts weniger als mathematische Uebereinstimmung ihrer Richtung mit der Führungslinie des Beckens. Der Schädel des Kindes wird einfach in das Becken hineingepresst und muss dann seinen Weg aus demselben selbst finden.

Dieser Bedingung entspricht Tarnier's Zange durch die gelenkige Verbindung des Zugapparates mit dem den Schädel fassenden Theil des Instrumentes. Sein Forceps gestattet dem Schädel eine grössere Freiheit in der Bewegung durch das Becken, indem er durch die articulirte Insertion der Zugkraft ihm die Möglichkeit lässt, die Räumlichkeit des Beckens auch bei nicht ganz geeigneter Zugrichtung besser auszunützen, statt sich durch die eine Componente dorthin pressen zu lassen, wo er unüberwindlichen Widerstand findet.

Denken wir uns Tarnier's Forceps (Fig. 2) in jene Zeichnung (Fig. 1) eingelegt, so stellt Punkt $G$ das Gelenk dar, durch welches die "Tiges de traction" mit den „Branches de préhension" verbunden sind. Der Effect, den jene Componente $(A N)$ jetzt hat, ist ein ganz anderer, weit günstigerer. Es ändert sich der Winkel, welchen die Zugstangen mit den Zangengriffen bilden. Die letzteren heben sich, weil der Schädel sammt den mit ihm verbundenen Zangenlöffeln, während er dem Zuge in der Becken-

1) Annales de Gynécologie 1877.

2) „Die Achsenzugzangen mit besonderer Berücksichtigung der Tarnier'schen Zangen." Stuttgart 1881. 
achse folgt, sich derart um seine quer im Becken liegende Achse drehen kann, dass ein grösserer Theil seiner hinteren Fläche in die geräumige Aushöhlung des Kreuzbeines herabtritt.

Der Widerstand, den die Componente $A N$ an der vorderen Beckenwand findet, weist ihm diesen Weg, während er durch die Componente $A D$ nach abwärts gezogen wird. Und er kann ihm folgen, weil die löffelenden, die ihn gefasst halten, nicht eine starre Linie mit den ,Tiges de traction" bilden.

Fig. 2.

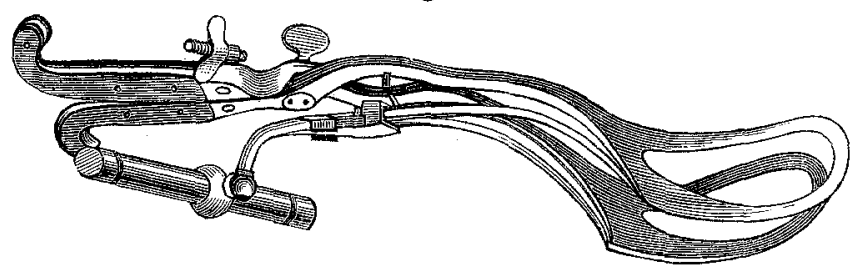

Tarnier's Modell 1878 (Progrès médical, Nr. 27). (Nach Fig. 4 von Simpson's Monographie.)

So wird der Schädel während jeder neuen Traction immer jene Stellung einnehmen können, in welcher er von Seite der Beckenwand dem geringeren Widerstande begegnet. Das Falsche einer ungünstigen Zugrichtung kann sich bis zu einer gewissen Grenze so von selbst corrigiren. Vermöge der gelenkigen Verbindung der Löffel mit dem Zugapparate wird die Richtung, in welcher gezogen wird, nicht dem Schädel zur Fortbewegung aufgezwungen wie bei Operationen mit dem gewöhnlichen starren Forceps. Zieht man die Löffel und mit ihnen den Schädel gegen die vordere Beckenwand, so können sie in dieser Richtung nicht folgen, treten aber mit dem Schädel dorthin, wo sie Raum zur Vorbewegung finden und ändern dabei nur ihre Stellung gegen die Zugstangen. Mit anderen Worten: die Fortbewegung des Schädels wird minder abhängig von der Richtung der Tractionen.

Dieser freiere Spielraum, den Tarnier's Forceps den Bewegungen des Schädels während der Traction im Gegensatze zur gewöhnlichen Zange gestattet, ist das wesentliche Moment, welchem wir den von keiner Seite bestrittenen notorisch geringeren Kraftaufwand bei Operationen mit seiner Zange verdanken. Nicht die Dammkrümmung, vermöge welcher der Zug ohne übermässiges Senken der Griffe doch immer in der Beckenachse möglich sein soll, und auch nicht die "Aiguille indicatrice" sind die Hauptsache an Tarnier's Zangenmodification, sondern die be- 
wegliche Verbindung jenes Theiles der Zange, an welchem gezogen wird mit den Löffeln, welche den Schädel fassen.

Wenn man aber nun Tarnier's Constructionen betrachtet, so müssen manche Inconvenienzen auffallen, die seine Art der Ausfïhrung des Principes der beweglichen Insertion der Zugkraft mit sich bringt.

Seine Zange besteht, da er nicht an den Griffen zieht, sondern eine eigene Zugvorrichtung anbringt, aus zwei gleichwerthigen Apparaten, den Branches de préhension und dem Tracteur, deren jeder grossen Anforderungen zu entsprechen hat und daher nicht nebensächlich ausgeführt sein kann, sondern sehr genau gearbeitet sein muss. Dadurch complicirt sich das Instrument gegenüber der einfachen Zange schon sehr. Der eine Theil, den die Branches de préhension bilden, hat sogar eine zweifache widersprechende Aufgabe. Sie müssen die Löffel am Schädel geschlossen erhalten und so den Angriff der Zange am Schädel sichern, da dies nicht mehr wie bei dem ,Forceps ordinaire“ die Hand des Operateurs besorgt, sondern eine Schraubenvorrichtung. Dazu müssen sie solid und stark gearbeitet sein. Gleichzeitig sollen sie aber auch als empfindliche ,Aiguille" dienen, welche frei in der Luft schwebend jede veränderte Stellung des Schädels im Beckenkanale anzuzeigen hat. Das sind zwei divergirende Anforderungen an ein und denselben Theil des Instrumentes, denen gleichzeitig nicht gut entsprochen werden kann. Taugen die ,Branches“ für das eine, dann können sie nur unvollkommen dem anderen entsprechen.

Während die ersten von Tarnier construirten Zangen nur wenig Aehnlichkeit mit dem „Forceps ordinaire" haben, sondern sehr fremdartige Instrumente darstellen, nähert sich Tarnier in seinen späteren Modellen doch wieder mehr der Form des gewöhnlichen Forceps. Sein neuestes Instrument zeigt keine Dammkrümmung ${ }^{\mathbf{I}}$ ) der Griffe mehr und stellt im Wesentlichen wieder einen modificirten Levret'schen Forceps dar, in dessen untere Löffelrippen ein Zugapparat eingelenkt ist. An dem letzteren, den ,Tiges de traction", wird gezogen. Der übrige eigentliche Forceps ragt dabei, mit Ausnahme der Löffel, frei aus der Vulva,

1) Die Gründe, die ihn bestimmten, die Dammkrümmung der Griffe aufzugeben, schildert Tarnier nebst anderem im Juniheft der Annales de Gynécologie 1882: "Considérations sur le forceps." An dem Zugapparate, dem ,Tracteur mobile", ist die Dammkrümmung aber beibehalten. 
und bewegt sich während des Zuges als "Aiguille indicatrice" auf und nieder, welche dem Operateur die Richtung, in welcher er ziehen soll, nach dem jeweiligen Verhältnisse des Schädels zur Beckenachse anzeigt.

Diese Construction, durch welche ein doppelter Apparat erfordert wird, einer zur Fixation der Löffel und ein zweiter als Zugvorrichtung, hat infolge des Missverhältnisses dieser beiden Theile des Instrumentes mehrfache Mängel. Die „Branches de préhension" stellen eine sehr plumpe, lästige "Aiguille“ vor, welche durch ihr Gewicht auch auf die Bewegungen des Schädels nicht ohne hemmenden Einfluss ist. Der Halt der Zange am Schädel wird ganz aus der Hand gegeben und bleibt ausschliesslich einem Schraubenhebel anvertraut. Die beiden Spangen der „Tiges" müssen erst wieder durch einen ziemlich complicirten Apparat, der nicht immer prompt genug fungirt, mit dem Querbalken, an dem gezogen wird, in Verbindung gesetzt werden. Daraus entspringt eine gewisse Unverlässlichkeit und Umständlichkeit in der Handhabung, indem bald dieses bald jenes an dem vielgliederigen Instrumente versagt.

Dieselbe Construction zeigt Simpson's Axis-traction forceps (Fig. 3). Auch er stellt eine Zange mit an den Löffeln eingelenktem

Fig. 3.

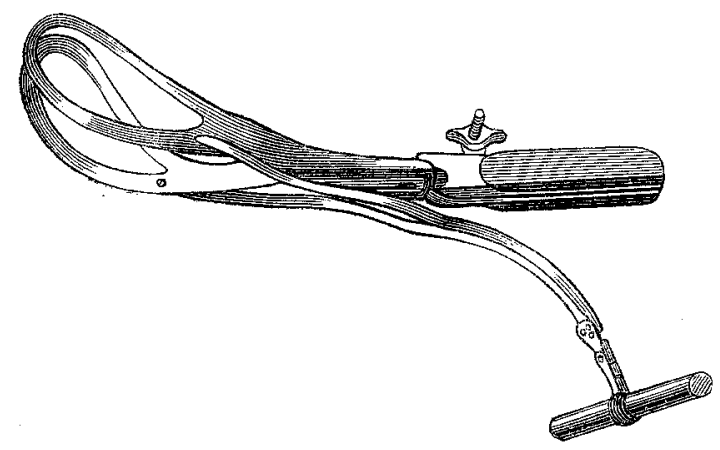

Simpson's Axis-traction forceps. (Nach Fig. 5 von Simpson's Monographie.)

stählernen Zugapparate vor. Nur dass wir hier statt des französischen einen englischen Forceps vor uns haben und der Verbindungsapparat der Zugstangen mit dem Querstabe ein anderer, aber noch unverlässlicherer ist. Das Instrument ist etwas einfacher zu handhaben als das Tarnier'sche: In einigen Fällen 
hat es an Prof. Gustav Braun's Klinik gut entsprochen. Aber in anderen Fällen wieder traten entschiedene Nachtheile zu Tage. Prof. Gustav Braun hat dem. Instrumente gleich von Anfang an weniger Vertrauen als dem französischen entgegengebracht. Die Zange ist, da sie doch für den höherstehenden Schädel in Verwendung gezogen werden soll, etwas zu klein und zu schwach gebaut. Während einer Operation brach das zu wenig solide Schloss, indem sich das eine vorspringende Blatt desselben abbog. Ein andermal sprang während einer Traction die eine Zugspange aus dem Verbindungsapparate am Querbalken. Und ein drittes Mal schnellte der Schraubenhebel, welcher die Griffe vereinigt, auf und die Zange verlor ihren Halt. Vor allem müsste diese Zange, wie schon Sänger angegeben, etwas grösser und stärker sein und die fixirende Schraube, welche zu nahe dem Schlosse liegt, mehr gegen das Griffende verlegt werden.

Die Fehlerquellen der Tarnier'schen und Simpson'schen Zangen liegen hauptsächlich, wie schon oben betont wurde, darin, dass sie doch noch zu sehr abweichen in Construction und Handhabung von unserem gewöhnlichen Forceps, daher die besten Eigenschaften desselben verlieren und zu so complicirten Apparaten werden. Ich habe mich deshalb bemüht, eine Achsenzugzange zu construiren, welche einfacher als die genannten Instrumente, sich möglichst an das Vorbild des „klassischen“ Forceps haltend, das Princip der gelenkigen Insertion der Zugkraft auf diesen überträgt und so die Bewegung des Schädels durch das Becken unabhängiger von der Richtung der Tractionen macht. 1)

Geleitet von diesem möglichst conservativen Bestreben versuchte ich jedes Zangenblatt unmittelbar hinter dem Fenster des Löffels durch ein Gelenk zu unterbrechen und es so einzurichten', dass die Griffe wie beim gewöhnlichen Forceps zugleich als Zugapparat und zur Fixirung der Löffel am Schädel dienen. Auf diese Weise liess sich dann die dem oberen Theile der Tarnier'schen Zangen entsprechende Partie des Instrumentes, welche bei letzteren mehr als Ballast denn als „Aiguille" erscheint, sehr reduciren, da sie nicht mehr wie bei Tarnier oder Simpson als „Branches de préhension“ zu fungiren hat. Dadurch, dass

1) Als ,Forceps souple à tractions indépendantes" veröffentlichte vor Kurzem im Lyon médical XXXVIII, Nr. 52, Poullet einen sehr complicirten Apparat, der aber auf ganz anderen als den hier entwickelten Principien beruht. 
die Griffe also wieder wie beim gewöhnlichen Forceps zum Geschlossenhalten der Zange und auch zum Ziehen gehören, wird, wenn man das Instrument mit dem Tarnier'schen vergleicht, dem oberen Theile der Zange, der dort als Branches de préhension dienen muss, die schwerere Aufgabe abgenommen, um derentwillen er bei Tarnier solid construirt und schwerfällig wurde. Ganz entbehrlich wird trotzdem dieser Theil des Instrumentes, der allein noch das plus desselben gegenüber dem einfachen gewöhnlichen Forceps darstellt, zwar nicht, auch wenn man auf ihn nicht als ,Aiguille indicatrice" reflectirt. Aber eine bedeutende Reduction und Vereinfachung desselben ergiebt sich.

Nach mehr als ein Jahr fortgesetzten Versuchen ergab sich also folgendes Modell. ${ }^{1}$ )

Durch ein starkes Plattengelenk, welches hinter den Fenstern das Zangenblatt bricht und sagittale Bewegung gestattet, wird die Veränderlichkeit des Winkels erzielt, unter welchem die Zugkraft an den Löffeln angreift. Die Fixirung der Löffel am Schädel geschieht dabei durch die im gewöhnlichen Schlosse vereinigten Griffe wie sonst mittels der Hand, welche an den Griffen zieht. Nur müssen die Löffel mit einander einigermaassen parallelisirt erhalten werden. Dies vermitteln zwei schlanke spornartige Fortsätze, welche von den oberen Löffelrippen nach aussen bis vor das Schloss geleitet werden und hier mit einander nur lose verbunden $\mathrm{zu}$ werden brauchen (Fig. 4).

Fig. 4.

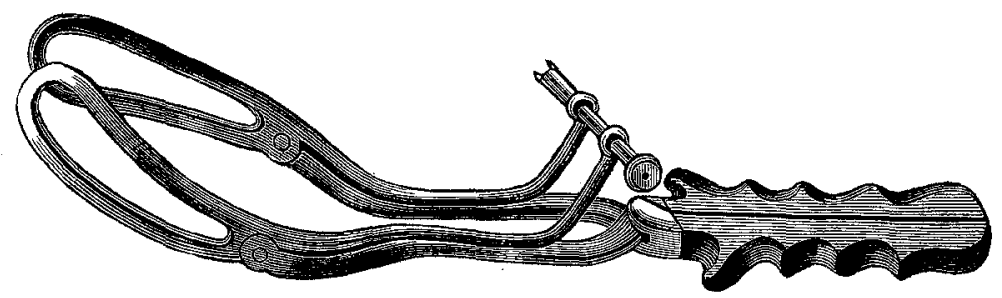

Die Zange mit ruhendem Gelenke.

Das Instrument stellt demnach einen etwas längeren gewöhnlichen Forceps mit starkem Schloss, wie er an den Wiener Kliniken für Operationen am hochstehenden Schädel gebräuchlich

1) Unser Instrumentenfabrikant J. Leiter hat mir mit gewohntem Interesse die nothwendigen Modelle präcis und sorgfältig ausgeführt, wofür ich ihm an dieser Stelle meinen Dank ausspreche. 
ist, vor, welcher durch folgende Einrichtung der Löffel zu einer Achsenzugzange wird.

Jedes Zangenblatt besteht aus zwei durch ein Gelenk mit einander verbundenen Hälften, dem Löffel und dem Griffe. Das obere Stück wird gebildet vom Löffel, welcher ein etwas kleineres Fenster von nur $10,5 \mathrm{~cm}$ Länge besitzt und hinter diesem an

Fig. 5.

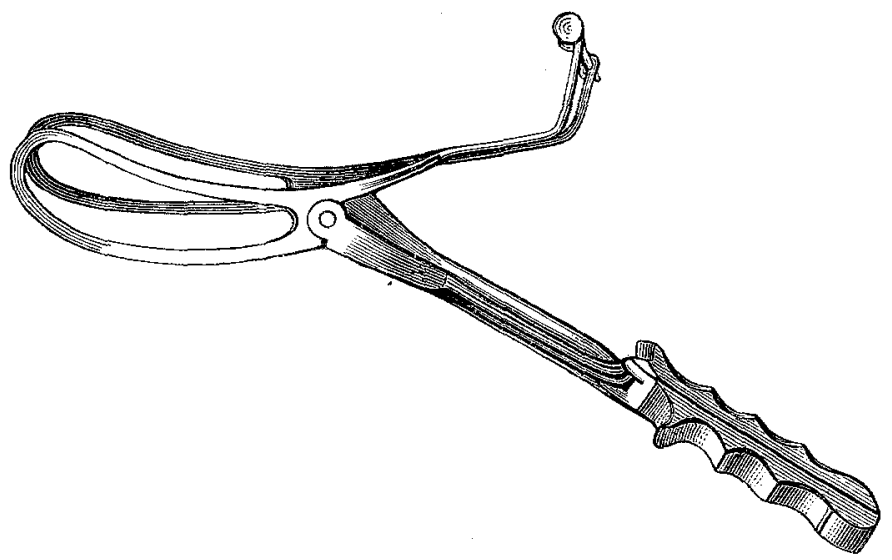

Die Zange mit geöffnetem Gelenke.

seiner äusseren Fläche mit einem abgestumpften halbkreisförmigen Rande übergeht in eine kreisrunde flache Aushöhlung mit vollkommen ebenem Boden. Die obere Rippe des Löffels setzt sich, genau der Krümmung des Zangenhalses folgend und sich an dessen oberen Rand anschmiegend, in einen runden dünnen Stahlstab auslaufend fort bis $3 \mathrm{~cm}$ vor den Schlosstheil. Hier krümmt sich dieser Fortsatz unter einem stumpfen Winkel nach aufwärts und endigt $5 \mathrm{~cm}$ über dieser Krümmung in einen kleinen sagittal gestellten Ring. Im Gegensatze zum gefensterten Theile, dem eigentlichen Löffel, kann man diesen Stahlstab den Löffelfortsatz nennen (Fig. 6).

Die untere Hälfte besteht aus dem übrigen unveränderten Theile des hinter dem Fenster abgesetzten Zangenblattes als dem Hals-Schlosstheile und dem Griffe. Indem das abgerundete Ende des Halstheiles eine kreisförmige Platte bildet, welche genau in die geschilderte Aushöhlung an der Aussenseite des Löffels passt, steht es mit diesem in gelenkiger Verbindung. Das Gelenk, welches diese beiden Stücke eines jeden Zangenblattes mit einander beweglich vereinigt, wird also gebildet durch zwei kreisrunde 
Platten von $2 \mathrm{~cm}$ Durchmesser, welche genau an einander passen und von einer starken Niete, die beide durchbohrt, zusammengehalten werden und um diese Niete als Achse rotiren. Alle Ränder und Kanten der beiden Gelenkplatten sind sorgfältig abgestumpft, so dass durch sie keinerlei Verletzungen der Weichtheile verursacht werden können. Weder an der äusseren noch

Fig. 6.

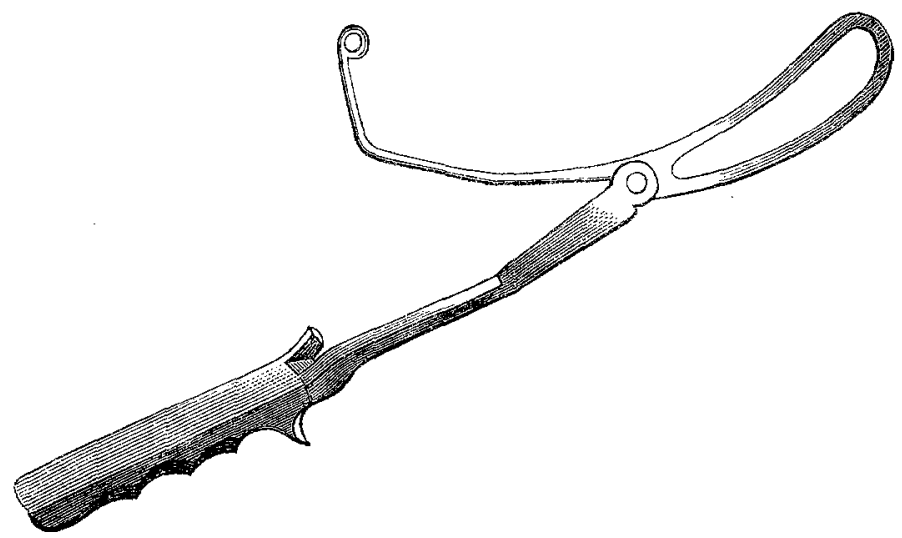

Das linke Blatt der Zange:

an der inneren Oberfläche des Löffels verursacht das Gelenk eine Niveauerhebung. Durch dieses Gelenk erhalten die Löffel die Fähigkeit, ibre Stellung zu den Griffen so weit zu verändern, dass sie mit diesen einen nach hinten offenen Winkel von circa 135 Graden bilden können.

Die beiden Platten des Gelenkes müssen durch die Achsenniete so mit einander verbunden sein, dass sie sich sehr leicht an einander verschieben können. So leicht beweglich muss das Gelenk sein, dass es, wenn man das Instrument frei hält und in verschiedene Neigung bringt, schon fungirt und sich die Stellung der Löffel zu den Griffen ändert. Die Niete, welche die Achse des Gelenkes bildet, ist genau $12,5 \mathrm{~cm}$ von der Löffelspitze entfernt.

Obwohl die Löffel durch das Gelenk Beweglichkeit in sagittaler $\left.{ }^{1}\right)$ Richtung erlangen, werden sie doch durch die im gewöhnlichen Schlosse vereinigten, von der ziehenden Hand umklambezogen.

1) Diese Bezeichnungen sind auf die Lage des Forceps im Becken , 
merten Griffe in vollkommen sicherer Lage am Schädel erhalten. Die beiden starken Stahlplatten, mittels welcher die im Gelenke verbundenen Stücke des Zangenblattes an einander um die Achsenniete rotiren, gestatten eben nur sagittale Bewegung, aber nicht, dass sich die Löffel in horizontaler Richtung so weit von einander entfernen, dass dadurch der Halt am Schädel unsicher würde. Wenn man das Instrument geschlossen in der Hand hält und das Gelenk spielen lässt, so bemerkt man zwar, dass sich die Löffelspitzen etwas von einander entfernen und der Abstand der oberen Rippen etwas vergrössert, wenn man die Löffel nach abwärts schlägt. Dabei nähern sich aber einander in gleichem Maasse die unteren Löffelrippen. Deshalh und da die Kopfkrümmung der Zange keine grosse ist, verliert durch diese geringen Schwankungen ${ }^{1}$ ) die Sicherheit des Haltes am Schädel nichts. Wohl aber wird vermöge der grösseren Motilität der Löffel der Schädel schonender gefasst.

Man kann sich davon leicht überzeugen, wenn man den Forceps an den Kopf eines neugeborenen Kindes anlegt. Nur eine incongruente divergirende Bewegung der beiden Löffel, so dass der eine vorn stehen bliebe, während der andere sich ganz nach rückwärts schlägt, würde der Zange den Halt am Schädel rauben. Eine solche ungleichartige Stellung der Löffel wird aber verhindert durch die spornartigen Fortsätze, welche sich über das Gelenk bis nahe vor das Schloss fortsetzen und hier durch einen kurzen Metallstift, der durch die ringförmigen Enden beider durchgesteckt wird, zu so weit es nöthig gleichartigen Bewegungen gezwungen werden. Durch diesen Stift und die Fortsätze werden die beiden Löffel immer so weit in paralleler Lage erhalten, dass sie nicht rom Schädel abgleiten können.

Wenn man die Ringe etwas breiter, röhrenförmiger anlegen und dem Caliber des Stiftes so anpassen würde, dass derselbe fest in ihnen steckt, so wäre die durch diese Einrichtung erzielte Parallelerhaltung der Löffel eine vollkommenere. Aber die Vorrichtung wäre schwerfälliger, die Einführung des Stiftes in die breiteren Ringe minder einfach. Wie ich mich durch vielfaches Experimentiren überzeugt habe, ist eine vollkommenere Paralle-

1) Die Kopfkrïmmung der Löffel, an welcher das Gelenk theilnimmt, verursacht dieselben. An einem aufmerksam gearbeiteten Instrumente sind sie aber so gering, dass sie nur vortheilhaft sind. 
lisirung der Löffel gar nicht nothwendig. Die durch die bestehende Einrichtung erreichte ist ganz genügend, um ein Abgleiten zu verhindern. Und je mehr Freiheit der Bewegung die Löffel haben, je mehr sie sich den speciellen Verhältnissen im gegebenen Falle anzupassen vermögen, desto schonender können sie ihre Aufgabe erfüllen. Sie dürfen nur nicht abgleiten, sie müssen den Schädel festhalten, aber sie sollen ihn nicht zwängen. Aus diesem Grunde, weil die freiere Beweglichkeit der Löffel nicht nur bei der zu erzielenden Kraftersparniss die geschilderte Rolle spielt, sondern auch ein schonenderes Fassen des Schädels vermittelt, wählte ich den ersteren Modus, die coordinirte Bewegung der Löffel zu sichern.

Der Metallstift, welcher durch die ringförmigen Enden der beiden Löffelfortsätze durchgeschoben wird, ist $9,5 \mathrm{~cm}$ lang, an dem einen Ende mit einem flachen Knopfe versehen, welcher sein Herausgleiten aus den Ringen verhindert. Zum selben Zwecke springen an dem anderen Ende zwei geknöpfte Federn widerhakenartig vor (Fig. 7).

Fig. 7.

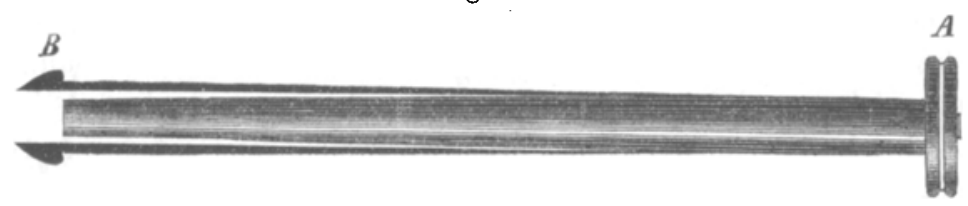

Stift, welcher die Bewegungen der Löffel coordinirt erhält.

Die geknöpften Enden dieser Federn sind so geformt, dass sie sich leicht und einfach, ohne dass man die Federn mit den Fingern zu comprimiren braucht, durch die weiten Ringe durchschieben lassen und das Herausfallen des Stiftes doch verhindern. Durch leichten raschen Zug an dem Ende $A$ lässt sich der Stift beim Abnehmen der Zange wieder aus den Ringen ziehen, da die Federn dann von selbst zurückweichen.

Die die Stellung der Löffel regulirenden spornartigen Fortsätze derselben krümmen sich unter einem stumpfen Winkel $3 \mathrm{~cm}$ vor dem Schlosse nach aufwärts, damit durch sie die Bewegungen der an den Busch'schen Haken der Griffe liegenden Hand nicht beeinträchtigt werden und damit sie beim Schliessen der Zange nicht hinderlich sind.

Der übrige untere Theil des Instrumentes, durch welchen es geschlossen und an welchem gezogen wird, bedarf keiner weiteren 
Schilderung, da er in nichts von dem gewöhnlichen an den Wiener Kliniken gebräuchlichen Forceps abweicht. Das Gelenk und die Fortsätze der Löffel mit dem Stifte sind alles, wodurch sich dieser Forceps ron dem gewöhnlichen unterscheidet.

Durch vielfache Versuche zuerst an der Leiche und später an der Gebärenden habe ich diese Construction geprüft und mich überzeugt, dass sie einfach und leicht $z u$ handhaben, ebenso be-quem und rasch anzulegen und zu schliessen als zu öffnen und abzunehmen ist. Der Halt am Schädel ist ein schonender und vollkommen sicherer ebenso wie bei dem gewöhnlichen Forceps. Ein Abgleiten kann nur unter Umständen stattfinden, unter welchen auch der gewöhnliche Forceps nicht hält. Man hat nur dieselben Cautelen zu beobachten, wie bei Handhabung des gewöhnlichen Forceps. Diesen Grundbedingungen entspricht das Instrument jedenfalls. Es ist weit einfacher als die bisherigen Constructionen, verlässlich und nicht gebrechlich. Dass es auch, wie ich glaube, jene Vortheile bietet, um derentwillen es construirt wurde, werden noch die Erfahrung und Versuche anderer Fachmänner bestätigen müssen.

Die Handhabung gestaltet sich folgendermaassen. Zuerst wird das linke Blatt eingeführt aus demselben Grunde, wie bei der gewöhnlichen Zange. Dabei fasst die linke Hand den Griff so, dass der Zeigefinger von aussen in den Winkel des Löffelfortsatzes zu liegen kommt, durch leichten Druck diesen an den Grifftheil anliegend erhält und auf diese Weise während der Einbringung des Löffels das Gelenk sperrt. Der Mittelfinger 'liegt an dem Busch'schen Haken, die beiden anderen Finger hinter demselben und der Daumen unterhalb des Schlosses. So gefasst wird das Zangenblatt nun wie das eines gewöhnlichen Forceps durch Schieben mit dem rechten Daumen und allmäliges Senken des Griffes eingefuhrt (Fig. 8).

Ebenso folgt dann das rechte Blatt. Beide Blätter werden dann leicht im Schlosse vereinigt, dabei die Griffe stark gesenkt und die aus der Vulva hervorragenden Löffelfortsätze an den Zangenhals niedergedrückt. Dann wird mit der linken Hand der Stift von rechts (i. e. vom rechten Löffel) nach links durch die ringförmigen Enden der Löffelfortsätze durchgeschoben.

Nun erst werden unter starkem Senken des Instrumentes und Andrücken der Löffelfortsätze an den Zangenhals die Griffe wie beim Operiren mit dem gewöhnlichen Forceps so weit anein- 
andergedrückt, als es nöthig ist, um die Löffel fixirt am Schädel zu erhalten. Dabei hat man sich ebenso wie bei anderen Forcepsoperationen vor zu starker Compression zu hüten, um den kindlichen Schädel unversehrt zu erhalten. Dass aber auch bei stärkerer Compression das Gelenk keine Einbusse an seiner Beweglichkeit erfährt, davon kann man sich leicht überzengen, wenn

Fig. 8.

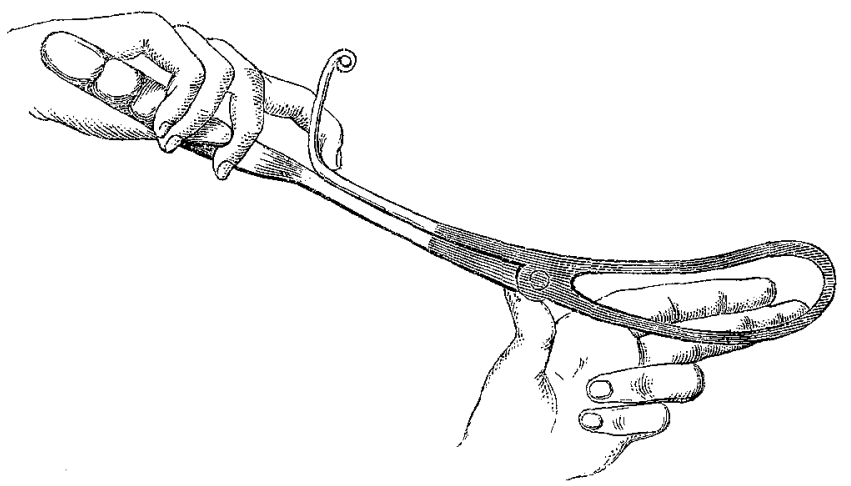

Einführung des rechten Zangenblattes. Zeige- und Mittelfinger der linken Hand, sowie die Spitze des Löffels sind in der Vagina liegend zu denken.

man den Schädel einer Kindesleiche unter stärkster Compreșsion in die Zange fasst und die Beweglichkeit des Gelenkes prüft.

Die Tractionen selbst kann man nun in der gewöbnlichen Weise mit einer Hand ausführen, während die andere Hand zur controlirenden Untersuchung frei bleibt oder zum Comprimiren der Griffenden, also zum sichereren Geschlossenhalten des Instrumentes verwendet wird, ganz so wie bei Handhabung des gewöhnlichen Forceps. Die ziehende Hand kommt dabei so wie sonst mit dem Mittelfinger in die Löffelkreuzung zu liegen, während die anderen Finger auf dem Busch'schen Haken ruhen.

Wenn sich während einer Traction der Stift, welcher die Stellung der Löffelfortsätze regulirt, etwas schief stellt, so hat dies nichts zu bedeuten, da die Incongruenz der Löffelstellung nie einen solchen Grad erreichen kann, dass sie gefährlich würde, und lässt sich überdies leicht corrigiren. Anfangs sollen die Tractionen stark nach abwärts gerichtet sein. Später, wenn sich die Löffelfortsätze mehr heben, kann horizontaler gezogen werden, und wenn der Schädel, am Beckenboden angelangt, in der Vulva sichtbar wird, müssen die Griffe ebenso wie beim Operiren mit dem gewöhnlichen Forceps gehoben werden. Wer eines Wegwei- 
sers für die Richtung, in der er ziehen soll, bedarf, kann sich von den Bewegungen der Löffelfortsätze ebenso leiten lassen, wie von der "Aiguille indicatrice" Tarnier"s.

Wie ich aber bereits oben auseinandergesetzt, bin ich nicht der Ansicht 'Tarnier's von der Wichtigkeit dessen, dass die Zugrichtung mit der Beckenachse so genau übereinstimme. Sondern es erscheint mir im Gegentheile als ein wesentlicher, von Tarnier nicht betonter Vorzug der sogenannten Achsenzugzangen (auch jener Tarnier's), dass sie die Bewegungen des Schädels durch den Beckenkanal unabhängiger machen von der Richtung, in der die Tractionen erfolgen, als dies beim gewöhnlichen starren Forceps der Fall ist.

Da der höher stehende, demnach nicht rotirte Schädel nicht in die Kopfkrümmung des Forceps passt, lassen sich anfangs die Griffenden beim Schliessen der Zange nicht ganz aneinander bringen, sondern stehen mehr oder weniger von einander ab und müssen während des Zuges entsprechend zusammengedrückt werden, damit der Schädel fest gefasst sei.

Bei dieser Zange geschieht diese unvermeidliche, für den Schädel immer bedenkliche Compression durch die Umklammerung der Griffe mit der Hand, wie beim gewöhnlichen Forceps, und kann daher viel vorsichtiger und schonender ausgeführt werden, weil durch das Gefühl das Maass derselben sich abschätzen lässt. Bei Tarnier's und verwandten Constructionen dagegen hält die Schraube die Griffe geschlossen. Der Rückschritt, der in dieser Einrichtung gegenüber dem gewöhnlichen Forceps liegt, ist augenfällig, sowie die Nachtheile, die aus ihm entspringen. Nach jeder Traction gestattet es die Einrichtung des geschilderten Instrumentes, dass die Zange ebenso, als ob man mit einem gewöhnlichen Forceps am nicht rotirten Schädel operiren würde, geöffnet werden kann. Man muss nur das Schloss lockern, indem man die Griffenden von einander mehr entfernt. Die durch den Stift vereinigten Löffelfortsätze sind dabei nicht im mindesten hinderlich. Man braucht nicht einmal den Stift aus denselben zu zichen, sondern kann ihn bis zum Abnehmen der Zange in seiner Lage belassen. Durch dieses Oeffnen des Instrumentes wird es den durch den Forceps gedrückten Geweben des Schädels möglich, sich während der Tractionspausen wieder vom Drucke, dem sie ausgesetzt waren, zu erholen. Der Druck, da er nicht continuirlich wirkt, wird dadurch minder gefährlich. Anderer- 
seits wird der durch die Traction etwas herabgerückte Schädel in der geöffneten Zange nicht an seiner Rotation behindert. Nach mehreren Tractionen, weun der Schädel am Beckenboden angelangt ist, wird man aus dem nun vollständigen Aneinanderpassen der Griffe beim erneuten Schliessen der Zange ersehen, dass der Schädel sich bereits rotirt habe.

Beim Schliessen der Zange nach einer jeden Tractionspause hat man nur die Griffe wieder stark zu senken und die Löffelfortsätze an die Zange anzudrücken, damit die Löffel den Schädel richtig fassen.

Die Wirksamkeit der Tractionen ist nach meiner Erfahrung eine eminente und dabei der Kraftaufwand ein so mässiger, dass stets die genaueste Ueberwachung des Fortganges der Operation möglich ist. Unter anderen gelang es z. B. bei einer Primipara mit plattem Becken von $8,5 \mathrm{~cm}$ Conjugata vera nach 47 stündiger Wehendauer und 13 Stunden nach dem. Blasensprunge, als der Schädel des $3300 \mathrm{~g}$ schweren Kindes erst im Beckeneingange fixirt, mit seinem Centrum noch nicht im Niveau desselben sich befand, auf drei ganz mässige Tractionen denselben soweit herabzubringen, dass die Zange abgenommen und der Schädel mit dem Ritgen'schen Handgriffe über den Damm gebrächt werden konnte. (Prot.Nr. 1463, 1882.)

Ist der Schädel nach mehreren Tractionen bis in die Vulva gebracht, dann hat der Forceps seine Schuldigkeit gethan und soll abgenommen werden. Der Dammschutz und die vollständige Entwickelung des Schädels wird nach Ritgen durchgeführt. Das Abnehmen der Zange unterliegt gar keinen Schwierigkeiten und kann mit der Präcision und Schnelligkeit geschehen, welche in dem kritischen Momente geboten sind, in welchem sich bereits der Damm spannt und beide Hände zum Dammschutz nöthig werden.

Nachdem der Mittelfinger in das Rectum eingeführt ist und durch dasselbe am Alveolarfortsatze des kindlichen Oberkiefers den Schädel fixirt, während der Daumen derselben Hand hart am Rande des Dammes den Schädel energissch gegen die Symphyse hinaufdrückt und so das Perinaeum entlastet, lässt die andere Hand die hocherhobenen Griffe los, bringt durch einen raschen Zug am Knopfende den Stift aus den Löffelfortsätzen und fördert dann durch Ziehen am Griffe und Ueberstürzen in die entgegengesetzte Inguinalgegend zuerst den rechten Löfel und 
dann den linken aus den Genitalien, ganz ebenso wie beim gewöhnlichen Forceps. Die ganze Procedur geht prompt und ohne Zeitverlust in wenigen Secunden vor sich, und nun ist auch die zweite Hand zum Dammschutze disponibel.

Dieser ist durchaus nicht schwieriger durchzuführen als nach hohen Zangenoperationen überhaupt, wo, ob mit diesem oder dem gewöhnlichen Instrumente operirt worden, bei Primiparen stets die rasche Durchleitung des Schädels durch die bei hohem Kopfstande noch wenig vorbereitete Vagina die Gefahr von mehr weniger tiefgreifenden Vaginalrupturen ${ }^{1}$ ) bedingt, welche dann ihrerseits den Eintritt eines Dammrisses begünstigen. Auch in solchen Fällen vermag aber eine rechtzeitige Vornahme der Episiotomie dem Eintreten einer grösseren Dammruptur vorzubeugen. Stellt sich während der Operation eine der gewissen Schwierigkeiten ein, welche überhaupt bei Zangenoperationen mitunter vorkommen, so kann man denselben in gleicher Weise begegnen wie bei Verwendung des gewöhnlichen Forceps.

Gelingt zum Beispiel das Einführen des einen Zangenblattes in der einen Seite nicht leicht, so versucht man es rückwärts hinaufzubringen, und bringt es erst dann an die seitliche Beckenwand. Oder manchmal kommt es auch bei Operationen mit dem gewöhnlichen Forceps vor, dass bei etwas spitzerem Arcus pubis oder sehr resistentem Perinaeum, wenn der Schädel bereits sichtbar ist, die stärksten Tractionen erfolglos bleiben, auch ohne dass gerade ein Missverhältniss zwisehen dem accommodationsfähigen Schädel und dem Beckenausgange bestünde.

Hier liegt bekanntlich die Ursache des unerwarteten Widerstandes darin, dass die Kopfkrümmung der Zange nicht unter dem Arcus pubis hervortreten kann, weil dieser spitzer als gewöhnlich, oder weil die grössere Resistenz des Beckenbodens das Instrument nicht genügend zurücktreten lässt und man daher nur die Zange gegen die Scham- und Sitzbeine anzieht, aber nicht den Schädel hervorzieht. Ebenso wie beim gewöhnlichen Forceps braucht man das Instrument dann nur etwas schief zu stellen, wenn man dieses Verhältniss constatirt hat, und die nächste Traction

1) Dass man für derartige Vaginalverletzungen nicht ohne weiteres das gebrauchte Instrument verantwortlich machen darf, zeigt das analoge Auftreten derselben bei manueller rascher Extraction von Steisslagen Erstgebärender. 
ist von Erfolg begleitet. Oder wenn sich beim Abnehmen der Zange ein Löffel an einem Ohre verfangen hat und nicht rasch genug befreien lässt, so kann man ihn ebenso wie den eines gewöhnlichen Forceps liegen lassen.

Ausser den oben geschilderten Eigenthümlichkeiten der Handhabung gelten überhaupt dieselben Regeln wie beim Gebrauche des gewöhnlichen Forceps.

Die Reinhaltung und Desinfection des Instrumentes ist leicht durchführbar. Man braucht es nur nach jedesmaligem Gebrauche gut abzuwaschen. Auch diejenige Stelle, die einem besonders scrupulösen Antiseptiker Sorgen machen könnte, das Gelenk, lässt sich durch Waschen leicht reinigen und durch öfteres Einfetten mit Vaseline, welches vor dem Gebrauche wiederholt wird, vollkommen sichern, ohne dass es zerlegt werden müsste. Wer das Gelenk dennoch als Brutstätte von Infectionskeimen fürchten würde, der müsste aus demselben Grunde auch den Gebrauch einer jeden Scheere zurückweisen, von anderen Instrumenten, zum Beispiel Perforatorien (sowobl scheeren - als trepanartigen), welche viel schwierigex und unvollkommener zu reinigen sind, ganz zu geschweigen.

Ein für den ersten Anblick ähnlicher, aber doch von dem geschilderten Instrumente ganz und gar verschiedener Forceps wurde im Jahre 1878 in Amerika von Vedder ${ }^{1}$ ) construirt, hat aber (wenigstens in Europa) keine weitere Beachtung gefunden.

Ausgehend von dem Vergleiche des Forceps mit einem Paar künstlicher Hände, vermisste Vedder am gewöhnlichen Forceps die Beweglichkeit der Löffel nach auf- und abwärts (motion at

1) Medical Record. New-York, 23. March 1878. „An Improvement in the obstetrical forceps."

Diese nur drei Spalten lange, ganz eigenthümlich verfasste Abhandlung ist in einem für einen wissenschaftlichen Journalartikel befremdenden Tone gehalten. Thales von Milet, Morse's Telegraf, Bell's Telephon, Howe's Nähmaschine, Galen und Anaxagoras füllen eine ganze Spalte des Artikels. Die Hauptsache dagegen, eine Schilderung des Gelenkes, durch welches sich eben Vedder's Forceps von dem gewöhulichen unterscheidet, wird mit folgenden Worten umgangen und abgefertigt: ,The woodcut here given does not, unfortunately, show the simple mechanism by which this fixation is effected; it is sufficiently explanatory, however, to enable any member of the profession to comprehend at a glance, both the nature of the improved forceps and the advantages which must result from its employment." 
the wrists), die diesen Bewegungen der Hände im Handgelenke entspräche.

$\mathrm{Da}$ er dieser eine in seiner Abhandlung nicht weiter begründete Wichtigkeit beilegte und um den Forceps dem Vorbilde der Hände noch ähnlicher zu machen, construirte er den in Figur 9 abgebildeten Forceps.

Fig. 9.

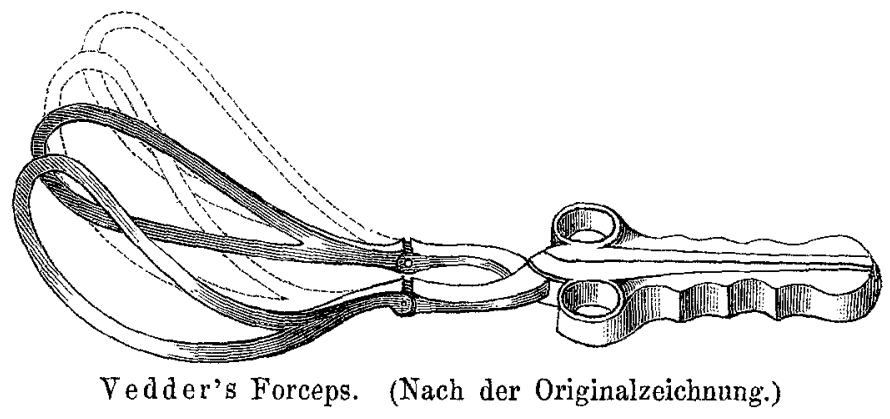

Derselbe hat ein Gelenk an jedem Zangenblatte in grosser Entfernung von der Löffelspitze im Zangenhalse, welches so eingerichtet ist, dass es während der Traction unbeweglich fixirt ist, nach der Traction aber dem Operateur gestattet, die Stellung der Griffe zu den Löffeln zu ändern und anders zu fixiren.

Da das Gelenk so weit von der Löffelspitze entfernt ist, wie ans der Zeichnung hervorgeht, dass es auch in ziemlicher Entfernung vom Schädel zu liegen kommt, und da es während der Tractionen nicht mobil, sondern fixirt ist, so ist der ganze Effect dieser Einrichtung nur der einer in den Tractionspausen modificirbaren Dammkrümmung des Forceps. Das Instrument hat daher in Princip und Ausfiihrung mit dem in Vorliegendem geschilderten Forceps gar nichts gemein. 